\title{
The formation of symmetrical glycosyl glycuronates by direct oxidation of partially protected methyl $\alpha$-D-glycopyranosides
}

\author{
Giovanna Cupone, Antonio De Nino, Renato Dalpozzo, Loredana Maiuolo, \\ Antonio Procopio, and Giovanni Sindona* \\ Dipartimento di Chimica, Università della Calabria, v. P. Bucci, cubo 15/c I \\ 87030 Arcavacata di Rende (CS)- Italy \\ E-mail: sindona@unical.it
}

Dedicated to Professor Domenico Spinelli on his $70^{\text {th }}$ birthday

(received 13 Oct 02; accepted 07 Dec 02; published on the web 15 Dec 02)

\begin{abstract}
The formation of symmetrical glycuronates by oxidation of methyl $\alpha$-D-2,3,4-tri-O-benzoyl glycopyranosides with pyridine dichlorochromate is described. A key intermediate in the process is represented by the initially formed aldehydes which react further through two competing reaction pathways leading to the glycals and to the symmetrical esters. The symmetrical glycuronates are formed by oxidation of the corresponding hemiacetal intermediates. The absolute configuration of the chiral centers at position 2-, 3-, and 4- of the glycopyranosides does not affect the two competing reaction paths.
\end{abstract}

Keywords: Gycopyranosides oxidation, symmetrical glycosyl glycuronates, sugar nitrones

\section{Introduction}

In the course of our studies concerning the synthesis of analogues of antiviral nucleoside drugs ${ }^{1}$ we planned the formation of chiral nitrones $\mathbf{5}$ from the fully protected glucopyranoside 1a through its stable 4,5- glucal, 3a, achievable through a previously reported ${ }^{2}$ procedure (Scheme, path $a$ ). The project aimed at exploiting new approaches to the enantioselective synthesis of isoxazolidino-nucleosides ${ }^{3}$ after the modification of the hexose ring through conventional chemistry.

Methyl $\alpha$-D-2,3,4-tri-O-benzoyl glucopyranoside 1a was obtained in three steps, from the corresponding methyl $\alpha$-D-glucopyranoside, by tritylation in pyridine ( $75 \%$ yield) followed by benzoylation in the same solvent ( $88 \%$ yield) and final de-blocking with trifluoroacetic acid at $0^{\circ}$ C $(85 \%$ yield $)$. The corresponding $N$-benzyl nitrone 5 was obtained in satisfactory yield (Scheme). 

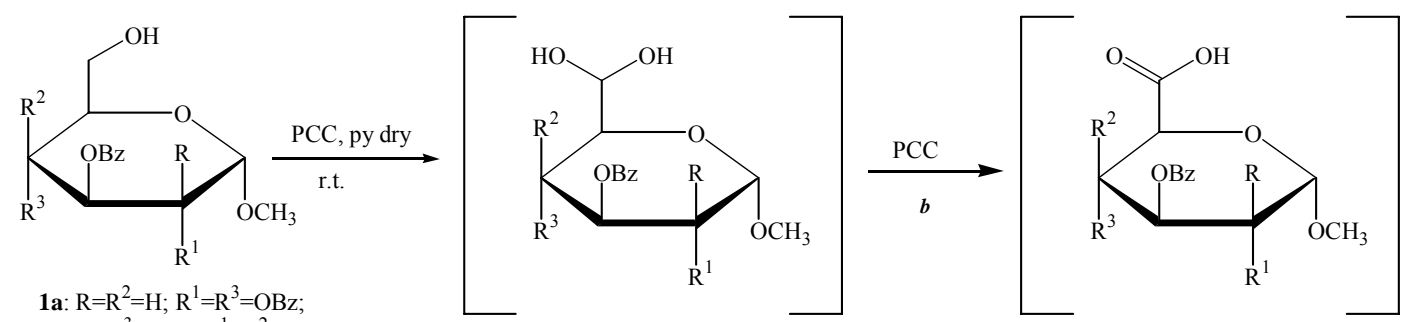

1a: $R=R^{2}=H ; R^{1}=R^{3}=O B z ;$
1b: $R=R^{3}=O B z ; R^{1}=R^{2}=H$

1c: $\mathrm{R}=\mathrm{R}^{3}=\mathrm{H} ; \mathrm{R}^{1}=\mathrm{R}^{2}=\mathrm{OBz}$;
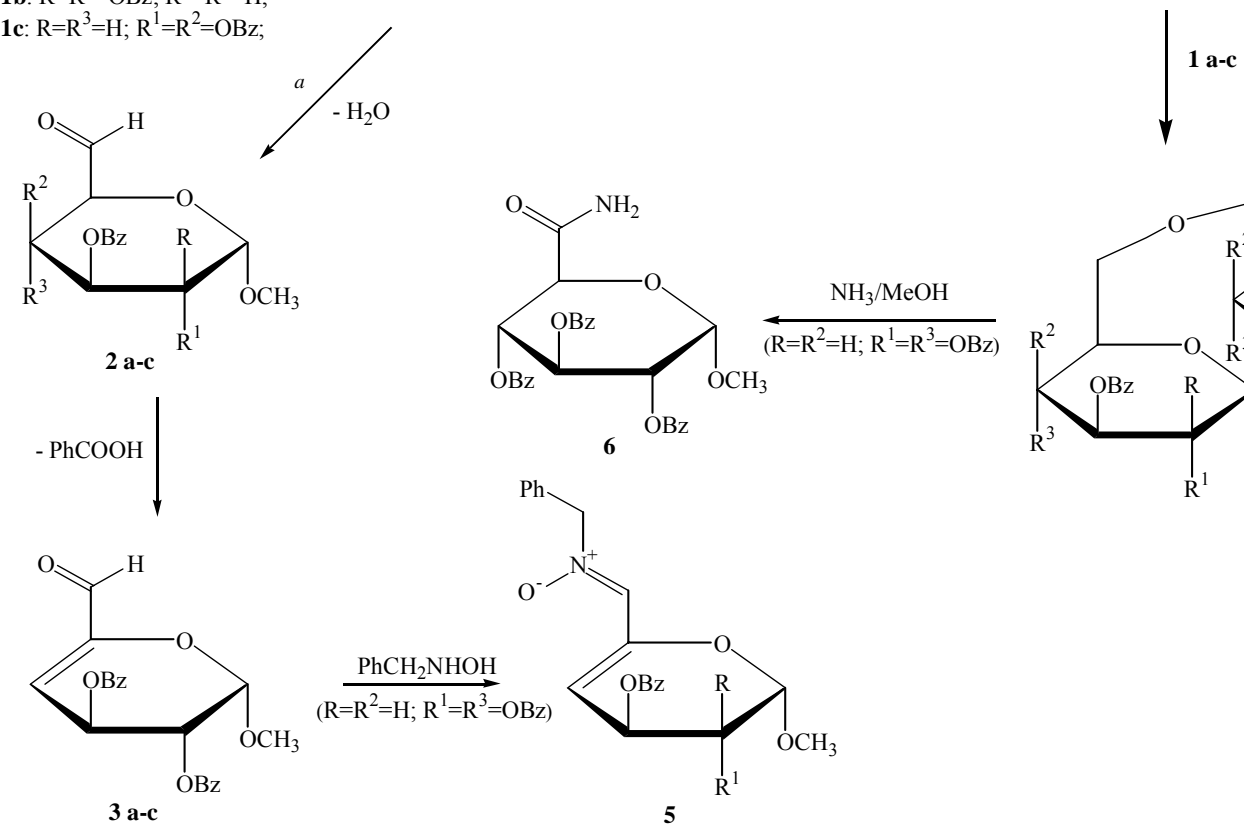

6

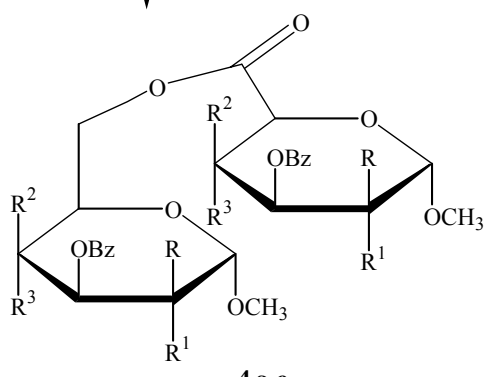

\section{Scheme}

The apparent limitation of the method was represented by the modest yields of the oxidation step (Scheme, path a) which were in contrast with a previous report ${ }^{2}$ on the oxidation of the same species protected at the positions 2, 3 and 4 with an acetyl group. However, a species having the structure of the glucuronate $4 \mathbf{a}$ was obtained in $45 \%$ isolated yield from the reaction mixture. The structural assignment was based on ${ }^{1} \mathrm{H}-\mathrm{NMR}$ and FAB- mass spectrometric data, and on the chemical information provided by the formation of methyl $\alpha$-D-glucuronamide $\mathbf{6}$ after conventional ammonia/methanol de-blocking (Scheme).

Table. Isolated yields (\%) of compounds $3 \mathbf{a}-\mathbf{c}$ and $\mathbf{4 a - c}$

\begin{tabular}{lll}
\hline Reagents & Aldehyde & Ester \\
\hline 1a & 3a (40) & 4a (45) \\
1b & 3b (40) & 4b (47) \\
1c & 3c (38) & 4c (42) \\
\hline
\end{tabular}


The generalization of this peculiar oxidation process was checked with other glycopyranosides under the same experimental conditions. Manno- (1b) and galacto-pyranosides

(1c) afforded the glycuronates $4 \mathbf{b}$ and 4c, with 47 and $42 \%$ yields, respectively, together with the glycals $\mathbf{3 b}, \mathbf{c}$ (Table).

From the data reported in the table it can be assumed that the formation of the ester (Scheme, path $b$ ) is an independent process competing with the formation of the $\alpha, \beta$-unsaturated aldehyde moiety of the glycals (Scheme, path $a$ ). Whatever the mechanism, in fact, no unsaturated glucuronides were formed. The elimination of a benzoic acid unit from positions -4 and -5 of the investigated glyco-pyranosides does not depend on the absolute configuration of the chiral centers involved in the process. It is, in fact, taken by the galacto- and gluco- isomers, affording the same glycal. The formation of the symmetrical esters is probably owing to the oxidation of intermediate hemiacetals originating from the interaction of the unreacted glycopyranosides 1a-c with the primarily formed aldehydes (Scheme).

To the best of our knowledge, the formation of symmetric glycuronides by oxidation of the corresponding glycopyranoside has never been described before, whereas it has been shown ${ }^{4}$ that even in the case of simple cyclic or acyclic alcohols, the reaction sequence alcohol $\rightarrow$ aldehyde $\rightarrow$ hemiacetal $\rightarrow$ symmetrical ester was preferred to the commonly accepted oxidation of alcohols to carboxylic acids, followed by esterification.

This reaction pathway, which is not usually recognized, can be in operation as a competitive process in the oxidation of glycopyranosides to 4,5-glycals and represents a valuable one-pot alternative to the conventional access to symmetrical glycopyranoside uronates. Moreover, the oxidation of the starting compounds proceeds with excellent isolated yields.

\section{Experimental Section}

General Procedures. NMR spectra were measured at $300 \mathrm{MHz}$; coupling constants, J, are in Hz. FAB-MS spectra were obtained in 3-nitrobenzyl alcohol matrix ( $m$-NBA).

Methyl $\alpha$-D-2,3,4-tri-O-benzoylglycopyranosides (1a-c) $(0.198 \mathrm{~g} ; 0.39 \mathrm{mmol})$ and pyridinium chlorochromate $(\mathrm{PCC})(0.13 \mathrm{~g} ; 0.59 \mathrm{mmol})$ were placed in $15 \mathrm{ml}$ of dry $\mathrm{CH}_{2} \mathrm{Cl}_{2}$ under dry nitrogen. Molecular sieves were added to this suspension. The reaction mixture was stirred for a week. Then $10 \mathrm{ml}$ of diethyl ether was added and the suspension was filtered through a column of silica gel. The colorless filtrate was concentrated in vacuo and the residue purified by flash chromatography (petroleum, b.p. $40-60{ }^{\circ} \mathrm{C} / \mathrm{Et}_{2} \mathrm{O}, 9: 1 \mathrm{v} / \mathrm{v}$ ). Two products (3ac and $\mathbf{4 a - c )}$ were isolated.

3a. $\delta_{\mathrm{H}}\left(\mathrm{CDCl}_{3}\right): 3.56\left(\mathrm{~s}, 3 \mathrm{H}, \mathrm{OCH}_{3}\right) ; 5.42\left(\mathrm{~d}, 1 \mathrm{H}, \mathrm{H}_{1}, \mathrm{~J}_{1,2}=2.4\right) ; 5.61\left(\mathrm{dd}, 1 \mathrm{H}, \mathrm{H}_{2}, \mathrm{~J}_{2,1}=2.4\right.$, $\left.\mathrm{J}_{2,3}=8.4\right) ; 6.08\left(\mathrm{~d}, 1 \mathrm{H}, \mathrm{H}_{4}, \mathrm{~J}_{4,3}=2.8\right) ; 6.19\left(\mathrm{dd}, 1 \mathrm{H}, \mathrm{H}_{3}, \mathrm{~J}_{3,2}=8.4, \mathrm{~J}_{3,4}=2.8\right) ; 8.10-7.30$ (m, 10H, Ar); 9.31 (s 1H, CHO); FAB-MS (+), m-NBA m/z: $383[\mathrm{M}+\mathrm{H}]^{+}(13 \%) ; 261[\mathrm{M}-\mathrm{BzOH}]^{+}(100 \%)$. Anal. Calcd. for $\mathrm{C}_{21} \mathrm{H}_{18} \mathrm{O}_{7}$; C 65.96, $\mathrm{H} 4.74$, O 29.29: found; $\mathrm{C} 66.28 \mathrm{H} \mathrm{4.26 \%}$. 
3b. $\delta_{\mathrm{H}}\left(\mathrm{CDCl}_{3}\right): 3.60\left(\mathrm{~s}, 3 \mathrm{H}, \mathrm{OCH}_{3}\right) ; 5.34\left(\mathrm{~d}, 1 \mathrm{H}, \mathrm{J}_{1,2}=2.7\right) ; 5.70\left(\mathrm{ddd}, 1 \mathrm{H}, \mathrm{H}_{2}, \mathrm{~J}_{2,1}=2.7, \mathrm{~J}_{2,3}=2.3\right.$, $\left.\mathrm{J}_{2,4}=1.9\right) ; 6.04$ (dd, $\left.1 \mathrm{H}, \mathrm{H}_{4}, \mathrm{~J}_{4,2}=1.9, \mathrm{~J}_{4,3}=4.6\right) ; 6.09$ (dd, $\left.1 \mathrm{H}, \mathrm{H}_{3}, \mathrm{~J}_{3,2}=2.3, \mathrm{~J}_{3,4}=4.6\right) ; 8.05-7.34$ (m, 10H, Ar); 9.31 (s 1H, CHO); FAB-MS (+), m-NBA m/z: $383[\mathrm{M}+\mathrm{H}]^{+}(34 \%) ; 261[\mathrm{M}-\mathrm{BzOH}]^{+}$ (100\%). Anal. Calcd. for $\mathrm{C}_{21} \mathrm{H}_{18} \mathrm{O}_{7} ; \mathrm{C}$ 65.96, $\mathrm{H} 4.74$, O 29.29: found; $\mathrm{C} 65.44, \mathrm{H} \mathrm{5.02 \%}$.

3c. $\delta_{\mathrm{H}}\left(\mathrm{CDCl}_{3}\right): 3.56\left(\mathrm{~s}, 3 \mathrm{H}, \mathrm{OCH}_{3}\right) ; 5.42\left(\mathrm{~d}, 1 \mathrm{H}, \mathrm{H}_{1}, \mathrm{~J}_{1,2}=2.8\right) ; 5.62\left(\mathrm{dd}, 1 \mathrm{H}, \mathrm{H}_{2}, \mathrm{~J}_{2,1}=2.8\right.$, $\left.\mathrm{J}_{2,3}=8.6\right) ; 6.08\left(\mathrm{~d}, 1 \mathrm{H}, \mathrm{H}_{4}, \mathrm{~J}_{4.3}=2.6\right) ; 6.19\left(\mathrm{dd}, 1 \mathrm{H}, \mathrm{H}_{3}, \mathrm{~J}_{3,2}=8.6, \mathrm{~J}_{3,4}=2.6\right) ; 8.15-7.35$ (m, 10H, Ar); 9.31 (s 1H, CHO); FAB-MS (+), m-NBA m/z: $383[\mathrm{M}+\mathrm{H}]^{+}(23 \%) ; 261[\mathrm{M}-\mathrm{BzOH}]^{+}(100 \%)$. Anal. Calcd. for $\mathrm{C}_{21} \mathrm{H}_{18} \mathrm{O}_{7}$; C 65.96, $\mathrm{H} 4.74$, O 29.29: found; C $65.83 \mathrm{H} \mathrm{5.28 \% .}$

4a. $\delta_{\mathrm{H}}\left(\mathrm{CDCl}_{3}\right): 3.20\left(\mathrm{~s}, 3 \mathrm{H}, \mathrm{OCH}_{3}\right) ; 3.45\left(\mathrm{~s}, 3 \mathrm{H}, \mathrm{OCH}_{3}\right) ; 4.25-4.30\left(\mathrm{~m}, 2 \mathrm{H}, \mathrm{H}_{5}, \mathrm{H}_{6 \mathrm{~A}}\right)$ ); $4.40(\mathrm{~m}$,

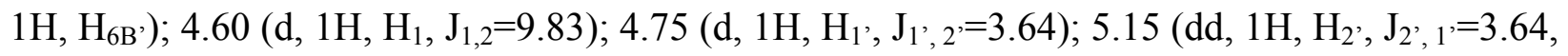
$\left.\mathrm{J}_{2^{\prime}, 3^{\prime}}=9.44\right) ; 5.30\left(\mathrm{~m}, 2 \mathrm{H}, \mathrm{H}_{5}+\mathrm{H}_{4}\right) ; 5.50\left(\mathrm{t}, 1 \mathrm{H}, \mathrm{H}_{4}, \mathrm{~J}_{4}, 3_{3}=\mathrm{J}_{4}, 5^{\prime}=9.44\right) ; 5.70\left(\mathrm{t}, 1 \mathrm{H}, \mathrm{H}_{2}, \mathrm{~J}_{2,3}=\mathrm{J}_{2}\right.$, $\left.{ }_{1}=9.83\right) ; 6.10\left(\mathrm{t}, 1 \mathrm{H}, \mathrm{H}_{3}, \mathrm{~J}_{3^{\prime}, 2},=\mathrm{J}_{3^{\prime}, 4},=9.44\right) ; 6.20\left(\mathrm{t}, 1 \mathrm{H}, \mathrm{H}_{3}, \mathrm{~J}_{3,2}=\mathrm{J}_{3,4}=9.83\right) ; 8.05-7.20 \mathrm{ppm}(\mathrm{m}$, $30 \mathrm{H}, \mathrm{Ar})$; FAB-MS (+), m-NBA m/z: $1031[\mathrm{M}+\mathrm{Na}]^{+}(50) \% ; 1009[\mathrm{M}+\mathrm{H}]^{+}(15 \%) ; 977[\mathrm{M}+\mathrm{H}-$ $\mathrm{MeOH}]^{+}$(100\%). Anal. Calcd. for $\mathrm{C}_{56} \mathrm{H}_{48} \mathrm{O}_{18} ; \mathrm{C}$ 66.66, H 4.80, O 28.54: found; $\mathrm{C} 67.11 \mathrm{H}$ $5.04 \%$.

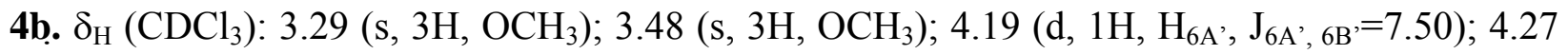
$\left(\mathrm{d}, 1 \mathrm{H}, \mathrm{H}_{6 \mathrm{~B}}, \mathrm{~J}_{6 \mathrm{~B}}, 6 \mathrm{~A}^{,}=7.50\right) ; 4.38\left(\mathrm{~d}, 1 \mathrm{H}, \mathrm{H}_{1}, \mathrm{~J}_{1^{\prime}, 2^{\prime}}=1.43\right) ; 4.42\left(\mathrm{~d}, 1 \mathrm{H}, \mathrm{H}_{5^{\prime}}, \mathrm{J}_{5^{\prime}, 4^{\prime}}=9.28\right) ; 4.54$ (dd, $\left.1 \mathrm{H}, \mathrm{H}_{4}, \mathrm{~J}_{4,3}=6.07, \mathrm{~J}_{4,5}=4.29\right) ; 5.07\left(\mathrm{~d}, 1 \mathrm{H}, \mathrm{H}_{1}, \mathrm{~J}_{1,2}=2.14\right) ; 5.52\left(\mathrm{dd}, 1 \mathrm{H}, \mathrm{H}_{2}, \mathrm{~J}_{2}, \mathrm{l}_{1}=1.43, \mathrm{~J}_{2}\right.$, $\left.3^{,}=3.21\right) ; 5.63\left(\mathrm{t}, 1 \mathrm{H}, \mathrm{H}_{2}, \mathrm{~J}_{2,1}=\mathrm{J}_{2,3}=2.14\right) ; 5.74\left(\mathrm{t}, 1 \mathrm{H}, \mathrm{H}_{4}, \mathrm{~J}_{4}, 3^{,}=\mathrm{J}_{4},{ }^{,}=9.28\right) ; 5.76\left(\mathrm{dd}, 1 \mathrm{H}, \mathrm{H}_{3}\right.$,

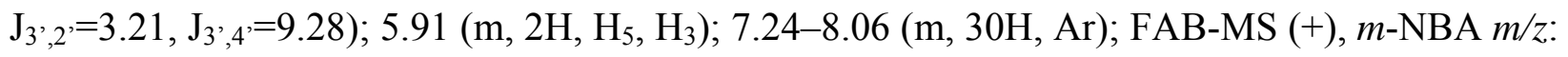
$1031[\mathrm{M}+\mathrm{Na}]^{+}(43 \%) ; 1009[\mathrm{M}+\mathrm{H}]^{+}(27 \%) ; 977[\mathrm{M}+\mathrm{H}-\mathrm{MeOH}]^{+}(100 \%)$. Anal. Calcd. for $\mathrm{C}_{56} \mathrm{H}_{48} \mathrm{O}_{18}$ : C 66.66, H 4.80, O 28.54; found; C $67.03 \mathrm{H} 4.22$

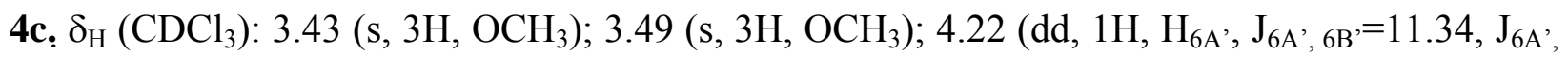

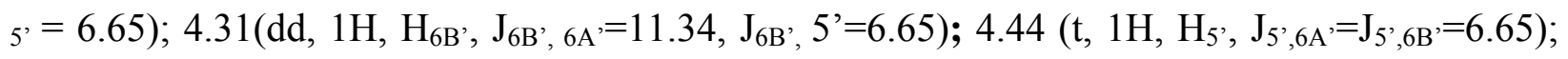
$4.90\left(\mathrm{~d}, 1 \mathrm{H}, \mathrm{H}_{5}, \mathrm{~J}_{5,4}=1.56\right) ; 5.22\left(\mathrm{~d}, 1 \mathrm{H}, \mathrm{H}_{1}, \mathrm{~J}_{1^{\prime}, 2}=3.52\right) ; 5.40\left(\mathrm{~d}, 1 \mathrm{H}, \mathrm{H}_{1}, \mathrm{~J}_{1,2}=3.52\right) ; 5.57$ (dd,

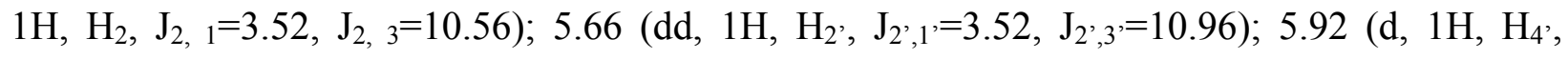

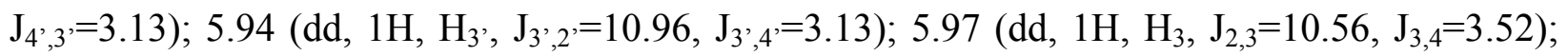
$6.20\left(\mathrm{dd}, 1 \mathrm{H}, \mathrm{H}_{4}, \mathrm{~J}_{4,3}=3.52, \mathrm{~J}_{4,5}=1.56\right) ; 8.02-7.01$ (m, 30H, Ar); FAB-MS (+), m-NBA m/z: 1031 $[\mathrm{M}+\mathrm{Na}]^{+}(30) \% ; 1009[\mathrm{M}+\mathrm{H}]^{+}(33) \% ; 977[\mathrm{M}+\mathrm{H}-\mathrm{MeOH}]^{+}(100 \%)$. Anal. Calcd. for $\mathrm{C}_{56} \mathrm{H}_{48} \mathrm{O}_{18}$; C 66.66, H 4.80, O 28.54: found, C 66.18 H 5.13\%.

5. $\delta_{\mathrm{H}}\left(\mathrm{CDCl}_{3}\right): 3.24\left(3 \mathrm{H}, \mathrm{s}, \mathrm{OCH}_{3}\right) ; 4.92\left(\mathrm{~s}, 2 \mathrm{H}, \mathrm{CH}_{2}\right) ; 5.20\left(\mathrm{~d}, 1 \mathrm{H}, \mathrm{H} 1, \mathrm{~J}_{1,2}=2.3\right) ; 5.48(\mathrm{dd}, 1 \mathrm{H}$, $\mathrm{H}_{2}, \mathrm{~J}_{2,1}=2.3, \mathrm{~J}_{2,3}=7.2$ ); $6.18\left(\mathrm{dd}, 1 \mathrm{H}, \mathrm{H}_{3}, \mathrm{~J}_{3,2}=7.3, \mathrm{~J}_{3,4}=4.0\right.$ ); $6.97\left(\mathrm{~s}, 1 \mathrm{H}, \mathrm{H}_{\mathrm{A}}\right) ; 7.21\left(\mathrm{~d}, 1 \mathrm{H}, \mathrm{H}_{4}\right.$, $\left.\mathrm{J}_{4,3}=4.1,\right) ; 7.26-8.00\left(\mathrm{~m}, 15 \mathrm{H}\right.$, Ar); FAB-MS (+), m-NBA m/z: $510[\mathrm{M}+\mathrm{Na}]^{+},(22 \%) ; 488$ $[\mathrm{M}+\mathrm{H}]^{+},(52 \%) ; 310$ (100\%). Anal. Calcd. for $\mathrm{C}_{28} \mathrm{H}_{25} \mathrm{NO}_{7} ; \mathrm{C} 68.98, \mathrm{H}$ 5.17, N 2.87, O 22.97: found; C 68.83, $\mathrm{H} 4.99, \mathrm{~N} 2.92 \%$.

6. ${ }^{5}$ FAB-MS (+), $m-\mathrm{NBA} \mathrm{C}_{7} \mathrm{H}_{13} \mathrm{NO}_{6}, \mathrm{~m} / \mathrm{z}: 230[\mathrm{M}+\mathrm{Na}]^{+}(33 \%) ; 208[\mathrm{M}+\mathrm{H}]^{+}(48 \%) ; 176[\mathrm{M}+\mathrm{H}-$ $\mathrm{MeOH}]^{+}(100 \%)$. 


\section{Acknowledgements}

This work was supported by a research grant of the University of Calabria.

\section{References}

1. (a) Leggio, A.; Liguori, A.; Procopio, A.; Siciliano, C.; Sindona, G. Tetrahedron Lett. 1996, 37, 1277. (b) Leggio, A.; Liguori, A.; Maiuolo, L.; Napoli, A.; Procopio, A.; Siciliano, C.; Sindona, G. J. Chem. Soc. Perkin Trans. 1 1997, 3097. (c) Colacino, E.; Converso, A.; De Nino, A.; Leggio, A.; Liguori, A.; Maiuolo, L.; Napoli, A.; Procopio, A.; Siciliano, C.; Sindona, G. Nucleosides \& Nucleotides 1999, 18, 581. (d) Dalpozzo, R. ; De Nino, A.; Maiuolo, L.; Procopio, A.; De Munno, G.; Sindona, G. Tetrahedron 2001, 17, 4035.

2. Cree, G. M.; Mackie, D. W.; Peril, A. S. Can. J. Chem. 1969, 47, 511.

3. (a) Pan, S.; Amankulor, N. M.; Zhao, K. Tetrahedron 1998, 54, 6587. (b) Converso, A.; Siciliano, C.; Sindona, G. In Targets in Heterocyclic Systems. Chemistry and Properties; Attanasi, O. A.; Spinelli, D. Eds; Italian Chemical Society: Rome, 1998; Vol. 2, 17.

4. Craig, J. C.; Horning, E. C. J. Org. Chem. 1960, 25, 2098.

5. Kovaik, V.; Mihalov, V.; Ková, P. Carbohydr. Res. 1979, 70, 239. 